\title{
Cross regulation of signaling pathways in gastrointestinal stromal tumor
}

\author{
YIJUN QI $^{1 *}$, WENDI ZHAO $^{2 *}$, ZHENGGUANG WANG $^{1}$, QINGSONG XIE $^{1}$, JING CAO $^{3}$ and XIANGLING MENG ${ }^{1}$ \\ ${ }^{1}$ Department of General Surgery, The First Affiliated Hospital of Anhui Medical University; ${ }^{2}$ Department of Pathology, \\ Anhui Medical University; ${ }^{3}$ Laboratory Medicine, The First Affiliated Hospital of \\ Anhui Medical University, Hefei, Anhui 230032, P.R. China
}

Received October 15, 2017; Accepted September 4, 2018

DOI: $10.3892 / \mathrm{ol} .2018 .9494$

\begin{abstract}
The Sonic hedgehog (Shh) signaling pathway may be interrelated with other signaling pathways, such as the phosphoinositide 3-kinase (PI3K) and mitogen-activated protein kinase (MAPK) pathways in gastrointestinal stromal tumor (GIST). The present study investigated the interaction among Shh, PI3K and MAPK signaling pathways in GIST cells. The expression of PI3K, MAPK and Shh signaling pathways in GIST-H1 cells were upregulated by endothelial growth factor (EGF) and recombinant Shh (N-shh) stimulation, and were downregulated by specific inhibitors of each signaling pathway. The proliferation rate of GIST-H1 cells were significantly increased under EGF or N-shh treatment $(\mathrm{P}<0.01)$. In addition, this effect was partially prevented by the pretreatment of the inhibitors of these signaling pathways. In summary, a cross regulation exists among the Shh, PI3K and MAPK signaling pathways in GIST-H-1 cells. The combined use of the inhibitors of these signaling pathways is a potentially novel option for GIST targeted therapy.
\end{abstract}

\section{Introduction}

Gastrointestinal stromal tumors (GIST) are the most common mesenchymal tumors of the gastrointestinal tract. The major genetic cause of GIST is the abnormal activation of c-KIT or platelet-derived growth factor receptor $\alpha$ (PDGFRA) due to acquired functional mutations (1). GIST patients are usually not sensitive to traditional radiotherapy or chemotherapy. Radical resection remains the treatment of choice, but $40-80 \%$

Correspondence to: Dr Xiangling Meng, Department of General Surgery, The First Affiliated Hospital of Anhui Medical University, 81 Meishan Road, Hefei, Anhui 230032, P.R. China

E-mail: xianglingmeng55@sina.com

*Contributed equally

Key words: gastrointestinal stromal tumor, Sonic hedgehog, phosphoinositide 3-kinase, mitogen-activated protein kinase, Imatinib-resistance of patients still develop recurrence or metastasis (2). Imatinib is a targeted therapy drug which inhibits tyrosine kinase (TK) activity. Imatinib has been used to treat Philadelphia chromosome-positive chronic myeloid leukemia (CML) and GIST patients who cannot undergo resection and/or already have metastasis. However, it has been reported that some patients did not respond to the drug, and some patients developed drug resistance.

Mutations in GIST lead to aberrant activation of TK in the absence of correspondingligands. Signaling pathways suchas the phosphoinositide 3-kinase (PI3K)/AKT and mitogen-activated protein kinase (MAPK) pathways are the most important signaling pathways that activate TK abnormally (3). These pathways may be involved in the mechanism contributing to the failure of Imatinib treatment (4). The Sonic hedgehog (Shh) pathway, on the other hand, is involved in the regulation of cell differentiation and proliferation. Previously it was considered to be highly conserved and plays a role only during embryonic development and tissue differentiation, and becomes inhibited in postnatal and adult tissues $(5,6)$. Zhao et al (7) first reported that inhibitors of Shh signaling pathway not only reduces the spread of wild-type BCR-ABL1 in CML, but also reduces the resistance to Imatinib in CML tumor cells. Subsequently, several other studies also suggested that the Shh pathway plays an important role in GIST (8-10). Therefore, it is worth studying if Shh pathway inhibitors may also be effective for Imatinib-resistant GIST. In addition, we speculate that PI3K, and MAPK signaling pathways may crosstalk with Shh for Shh inhibitor to act in Imatinib-resistant GIST. The study objective is to investigate the interaction among Shh, PI3K and MAPK pathways in GIST cells.

\section{Materials and methods}

Cells and major reagents. GIST-H1 cells were purchased from Shanghai Cell Bank, Chinese Academy of Sciences. HEGF and hN-SHH were procured from PeproTech and Sino Biological, respectively. Wortmannin and cyclopamine (CPN)-KAAD were obtained from Gene Operation.

PD98059 is a MAPK signaling pathway inhibitor commonly used in basic research. PD98059 can enter the cells through facilitated diffusion and specifically inhibit the ERK kinase MEK1, and ultimately inhibit the phosphorylation of 
ERK1/2 and block the MAPK signaling pathway. CPN is an isosteroid alkaloids. In vertebrates, the $\mathrm{SHH}$ signaling pathway is mainly composed of shh ligand, patched (PTC) receptor, smoothened (SMO) transmembrane protein, and nuclear transcription factor Gli (glioma-associated oncogene). Smo is an essential receptor for activating hedgehog signal transduction. Studies have confirmed that CPN can specifically antagonize Smo, so that Smo-dependent intracellular signal transduction of tumor gene transcription and expression was inhibited, thereby inhibiting the entire pathway activity. P110 $\beta$ subunit, $\mathrm{p} 85 \alpha$ subunit and the downstream molecules are all important members of PI3K signaling pathway. Blocking any one of these PI3K signaling pathways can be an option to block PI3K. Wortmannin is considered to be a highly selective inhibitor because it specifically binds only to the catalytic subunit P110 $\beta$ in PI3K.

The following substances and antibodies were used: MTT and mouse anti-tubulin antibody (Sigma-Aldrich; Merck KGaA, Darmstadt, Germany); rabbit anti-p-ERK1/2 antibody and rabbit anti-p-AKT antibody (Cell Signaling Technology, Inc., Danvers, MA, USA); rabbit anti-AKT antibody and rabbit anti-ERK1/2 antibody (BioWorld Technology, Inc., St. Louis Park, MN, USA); and PD 98059, mouse anti-caspase-3 antibody, and rabbit anti-Gli-1 antibody (Santa Cruz Biotechnology, Inc., Dallas, TX, USA).

Treatments. The GIST-H1 cells were induced by endothelial growth factor (EGF) and recombinant Shh (N-shh) through the treatments of blank control, CPN-KAAD, wortmannin, PD98059, CPN-KAAD+PD98059, and CPN-KAAD+wortmannin. The cells were collected after $48 \mathrm{~h}$, and the expression levels of p-AKT, AKT, p-ERK1/2, ERK1/2, and Gli-1 were detected via western blot.

Apoptosis test. One group was treated as follows: i) blank treatment, ii) blank treatment after $48 \mathrm{~h}$ starvation, iii) CPN-KAAD+wortmannin treatment after $48 \mathrm{~h}$ starvation, and iv) CPN-KAAD+wortmannin treatment after $48 \mathrm{~h}$ starvation. The other group was stimulated with $100 \mathrm{ng} / \mathrm{ml}$ EGF for 10 min with blank treatment, blank treatment after 48 h starvation, CPN-KAAD+wortmannin treatment, and CPN-KAAD+wortmannin treatment after $48 \mathrm{~h}$ starvation. The caspase-3 expression was determined through western blot.

Cell proliferation rate. The cells were divided into three groups: Group 1 was given blank treatment, blank treatment after 10 min CPN-KAAD, EGF (or N-Shh) stimulation, or CPN-KAAD treatment after 10 min EGF (or N-Shh) stimulation; group 2 was given PD98059 for $1 \mathrm{~h}$ with blank treatment, blank treatment after $10 \mathrm{~min}$ CPN-KAAD, EGF (or N-Shh) stimulation, or CPN-KAAD treatment after 10 min EGF (or $\mathrm{N}-\mathrm{Shh}$ ) stimulation; and group 3 was provided wortmannin for $1 \mathrm{~h}$ with blank treatment, blank treatment after $10 \mathrm{~min}$ CPN-KAAD, EGF (or N-Shh) stimulation, or CPN-KAAD treatment after 10 min EGF stimulation. Optical density was measured at $590 \mathrm{~nm}$, and each experiment was repeated twice.

Statistical analysis. SPSS 16.0 software was used for statistical analysis. The analytical methods used were the Student's t-test and chi-square test with $\mathrm{P}<0.05$ as significance level. In multi-group analysis, ANOVA followed by Tukey's test was used. In the cell growth curve, time was listed in the horizontal axis and light absorption value was assigned in the vertical axis.

\section{Results}

A number of MAPK, PI3K, and Shh signaling pathway inhibitors were used in the study. Wortmannin can initiate specific binding reactions with the catalytic subunit P110 $\beta$ in PI3K, which can inhibit PI3K activity. PD98059 is a MAPK signaling pathway inhibitor that inhibits the phosphorylation of ERK1/2 and blocks the transduction of the MAPK signaling pathway. CPN is an isosteroidal alkaloid that works specifically against Smo, which inhibits the transcription and expression of tumor genes that mediate the intracellular signal transduction of Smo and thus blocks the activity of the entire pathway. CPN-KAAD, a derivative of CPN, acts as a selective inhibitor of the Smo and hedgehog signaling pathways and is 10-20 times more active than $\mathrm{CPN}^{[2-3]}$.

Effects of EGF. Fig. 1 shows the relationship among PI3K, MAPK, and Shh signaling pathways under the action of EGF p-AKT and p-ERK expression. For both p-AKT and p-ERK, (1) The signal intensity of the EGF-treated control group was significantly higher than that of the non EGF-treated group $(\mathrm{P}<0.05)(2)$. The signal intensity between the wortmannin-, CPN-KAAD-, and wortmannin and CPN-KAAD co-treated groups, and the control group was significantly different $(\mathrm{P}<0.05)$. The signal intensity of the CPN-KAAD-, wortmannin-, and wortmannin+CPN-KAAD-treated groups was also lower than that of the EGF-treated control group $(\mathrm{P}<0.05$; Fig. 1A and B) (3). The signal intensity of the wortmannin-, PD98059-, and PD98059+CPN-KAAD-treated group was significantly lower than that of the control group $(\mathrm{P}<0.05)$. The signal intensity of the PD98059-, CPN-KAAD-, and CPN-KAAD+PD98059-treated groups was significantly lower than that of the EGF-treated control group $(\mathrm{P}<0.05$; Fig. 1C and D).

In Gli-1 expression, the signal intensity of the wortmannin-treated group was slightly lower than that of the control group $(\mathrm{P}<0.05)$. The signal intensity of the CPN-KAAD - and wortmannin+CPN-KAAD-treated groups was significantly lower than that of the control group $(\mathrm{P}<0.05)$. The signal intensity of the EGF-treated group was obviously higher than that of the non-EGF-treated group $(\mathrm{P}<0.05)$. In the EGF-treated group, the signal intensity of the inhibitor-treated group was obviously lower than that of the EGF-treated control group $(\mathrm{P}<0.05)$. Regardless of EGF treatment, the signal intensity of the co-treatment group was significantly lower than those of the wortmannin- and CPN-KAAD-treated group ( $\mathrm{P}<0.05$; Fig. $1 \mathrm{E}$ and $\mathrm{F})$. The signal intensity of the PD98059-treated group was lower than that of the control group $(\mathrm{P}<0.05)$, and the signal intensity of the CPN-KAAD- and PD98059+CPN-KAAD-treated groups was lower than that of the control group. The signal intensity of the EGF-treated group was significantly higher than that of the non-EGF-treated group $(\mathrm{P}<0.05)$. In the EGF-treated group, the signal intensity of the drug-treated group was significantly 
A

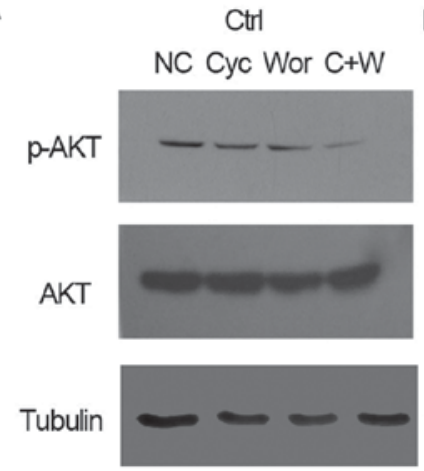

C

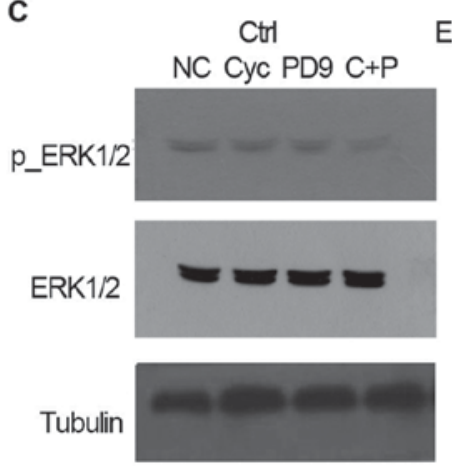

E



G

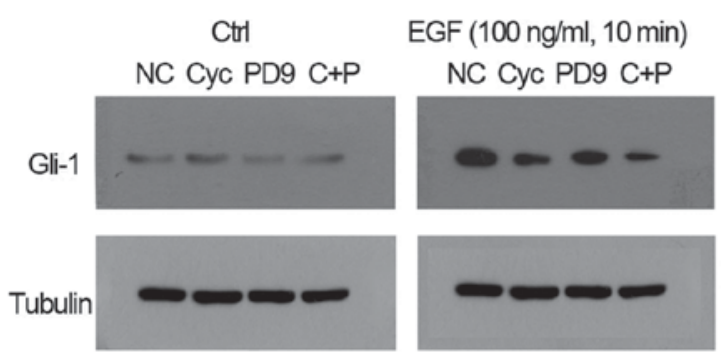

EGF (100 ng/ml, $10 \mathrm{~min})$
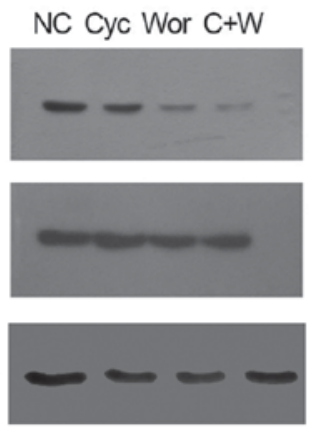

EGF (100 $\mathrm{ng} / \mathrm{ml}, 10 \mathrm{~min})$ NC Cyc PD9 C+P


EGF (100 $\mathrm{ng} / \mathrm{ml}, 10 \mathrm{~min})$<smiles>[I-]</smiles>

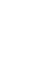

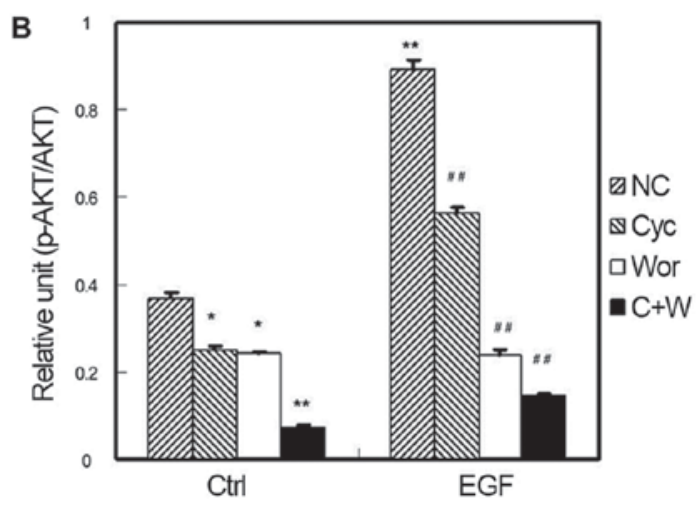

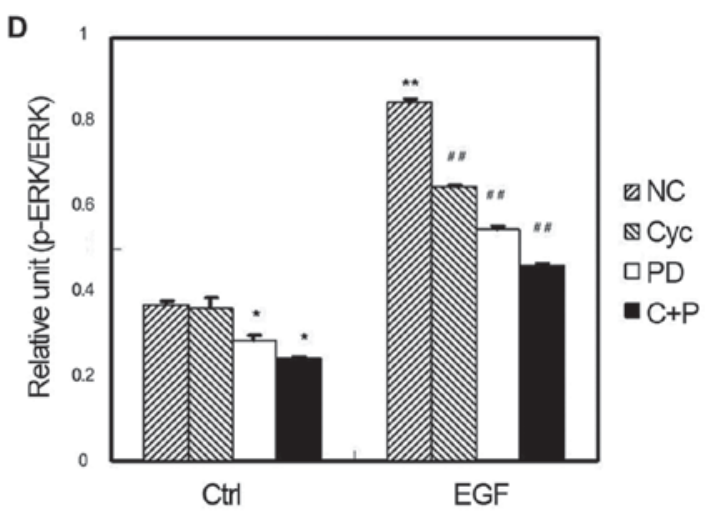

$\mathbf{F}$

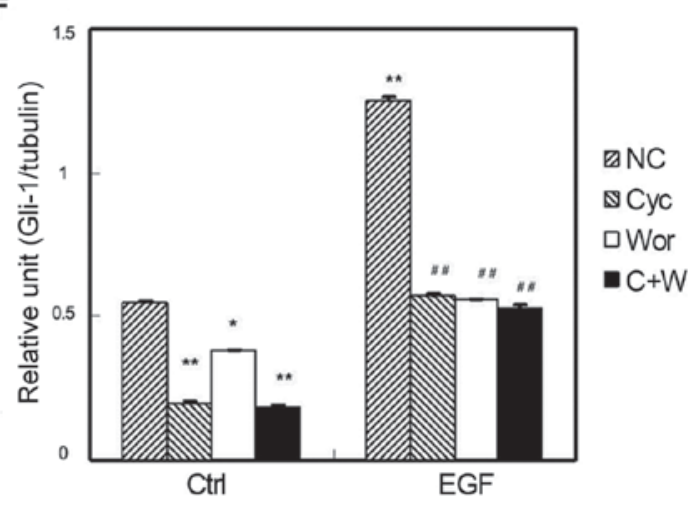

H



Figure 1. Effect of N-Shh on GIST-H1 cell signaling pathways. (A) Representative gel and quantification of (B) the effect of N-Shh on GIST-H1 cell AKT activation and the comparison of the effect from each treatment factor. (C) Representative gel and quantification of (D) the effect of N-Shh on GIST-H1 cell ERK activation and the comparison of the effect from each treatment factor. (E) Representative gel and quantification of (F) the effect of N-Shh on GIST-H1 cell Gli-1 expression and the comparison of the effect from each treatment factor. $(\mathrm{G})$ Representative gel and quantification of $(\mathrm{H})$ the effect of $\mathrm{N}$-Shh on GIST-H1 cell Gli-1 expression and the comparison of the effect from each treatment factor $\left(" \mathrm{P}<0.05,{ }^{* *} \mathrm{P}<0.01\right.$ vs. ctrl-NC; ${ }^{*} \mathrm{P}<0.05$, ${ }^{\# \#} \mathrm{P}<0.05$ vs. N-Shh-NC). $\mathrm{N}-\mathrm{Shh}$, recombinant Sonic hedgehog; GIST, gastrointestinal stromal tumor; EGF, endothelial growth factor; Gil-1, glioma-associated oncogene-1; NC-normal control, Cyc-cyclopamine, PD-PD98059, C+P-CPN-KAAD+PD98059, Wor-wortmannin, C+W- CPN-KAAD +wortmannin.

lower than that of the EGF-treated group $(\mathrm{P}<0.05)$. Regardless of the EGF treatment, the signal intensity of the co-treatment,
PD98059-, and CPN-KAAD-treated groups was significantly lower than that of the control group $(\mathrm{P}<0.05$; Fig. $1 \mathrm{G}$ and $\mathrm{H})$. 
A

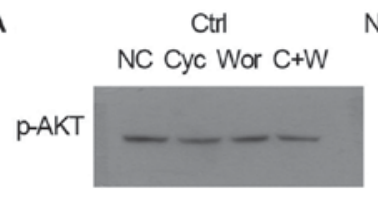

AKT

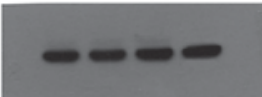

Tubulin

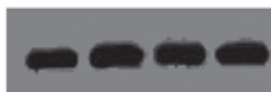

C
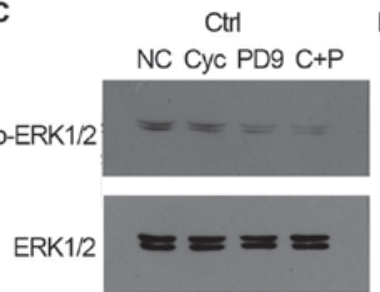

Tubulin

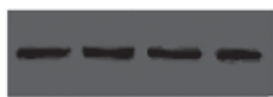

E

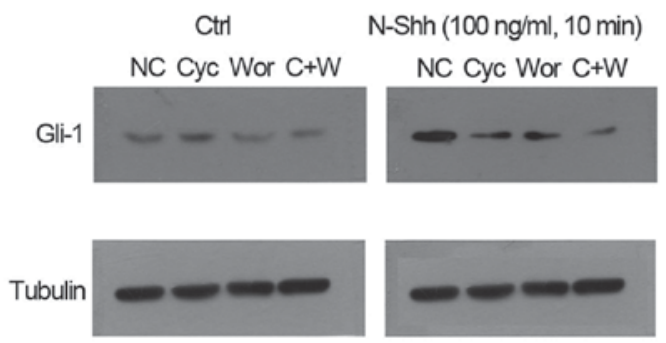

G

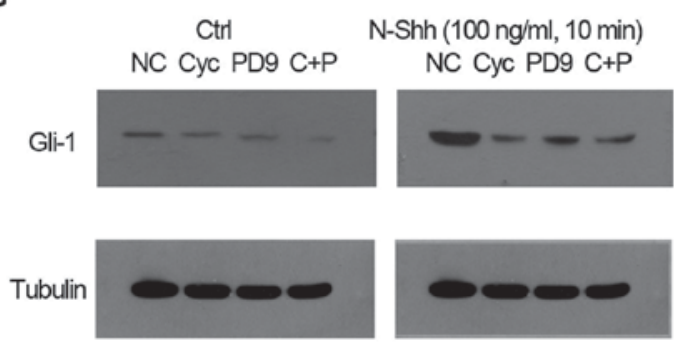

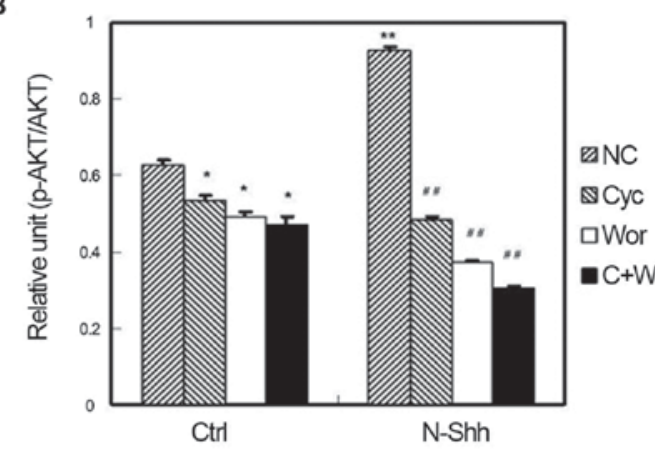

D

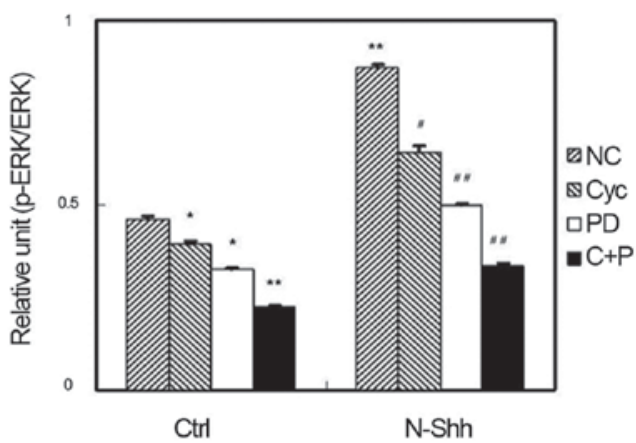

F

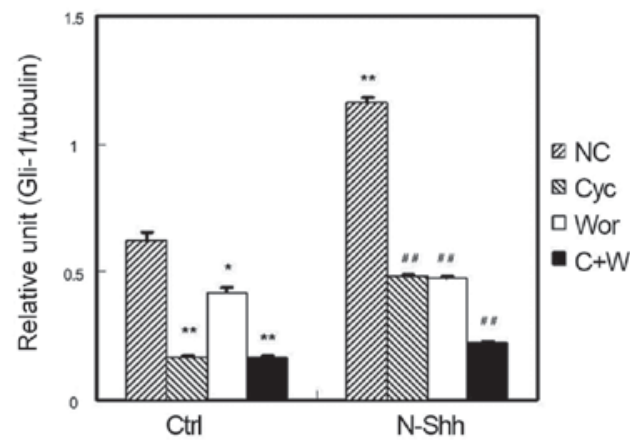

H

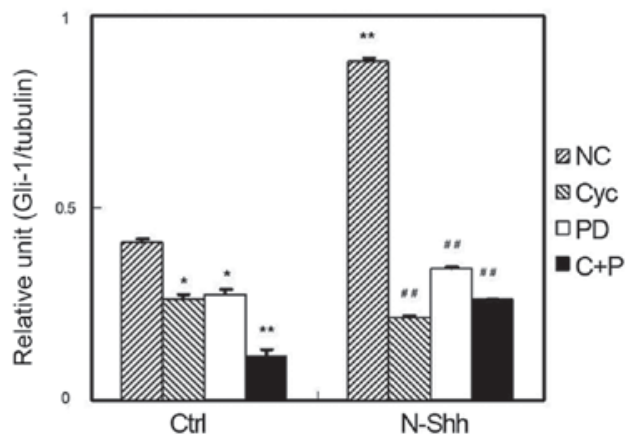

Figure 2. Effect of N-Shh on GIST-H1 cell signaling pathways. (A) Representative gel and quantification of (B) the effect of N-Shh on GIST-H1 cell AKT activation and the comparison of the effect from each treatment factor. (C) Representative gel and quantification of (D) the effect of N-Shh on GIST-H1 cell ERK activation and the comparison of the effect from each treatment factor. (E) Representative gel and quantification of (F) the effect of N-Shh on GIST-H1 cell Gli-1 expression and the comparison of the effect from each treatment factor. (G) Representative gel and quantification of (H) the effect of N-Shh on GIST-H1 cell Gli-1 expression and the comparison of the effect from each treatment factor $\left({ }^{*} \mathrm{P}<0.05,{ }^{* * *} \mathrm{P}<0.01\right.$ vs. ctrl-NC; ${ }^{\#} \mathrm{P}<0.05$, ${ }^{\# \#} \mathrm{P}<0.05$ vs. N-Shh-NC). N-Shh, recombinant Sonic hedgehog; GIST, gastrointestinal stromal tumor; EGF, endothelial growth factor; NC-normal control, Cyc-cyclopamine, PD-PD98059, C+P-CPN-KAAD+PD98059, Wor-wortmannin, C+W- CPN-KAAD+wortmannin.

Effects of $N$-Shh. Fig. 2 shows the relationship among PI3K, MAPK, and Shh signaling pathways under the action of N-Shh. In p-AKT expression, the signal intensity of the wortmannin-, CPN-KAAD-, and wortmannin+CPN-KAAD-treated groups was significantly lower than that of the control group $(\mathrm{P}<0.05)$. The signal intensity of the N-Shh-treated group was significantly higher than that of the control group $(\mathrm{P}<0.05)$. In the
$\mathrm{N}$-Shh-treated group, the signal intensity of the drug-treated group was significantly lower than that of the N-Shh-treated control group $(\mathrm{P}<0.05)$ (Fig. 2A and B). In p-ERK expression, the signal intensity of the PD98059- and CPN-KAAD-treated groups was lower than that of the control group $(\mathrm{P}<0.05)$. The signal intensity of the PD98059+CPN-KAAD-treated group was significantly higher than that of the control group 
A

$\begin{array}{lllll}\text { EFG } & - & - & - & - \\ \text { Sta } & - & + & + & + \\ \text { Cyc } & - & - & + & - \\ \text { Wor } & - & - & - & +\end{array}$

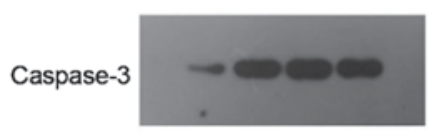

Tubulin

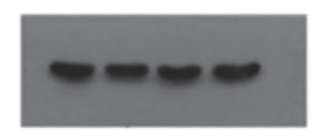

C
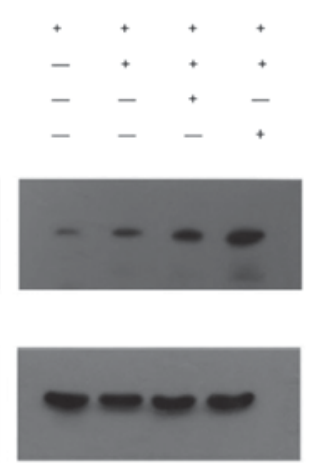

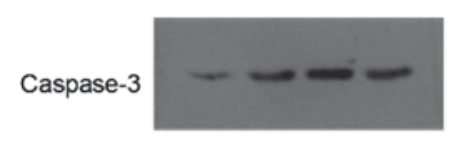

Tubulin

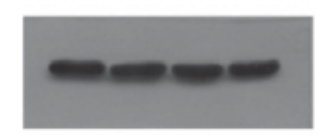

B
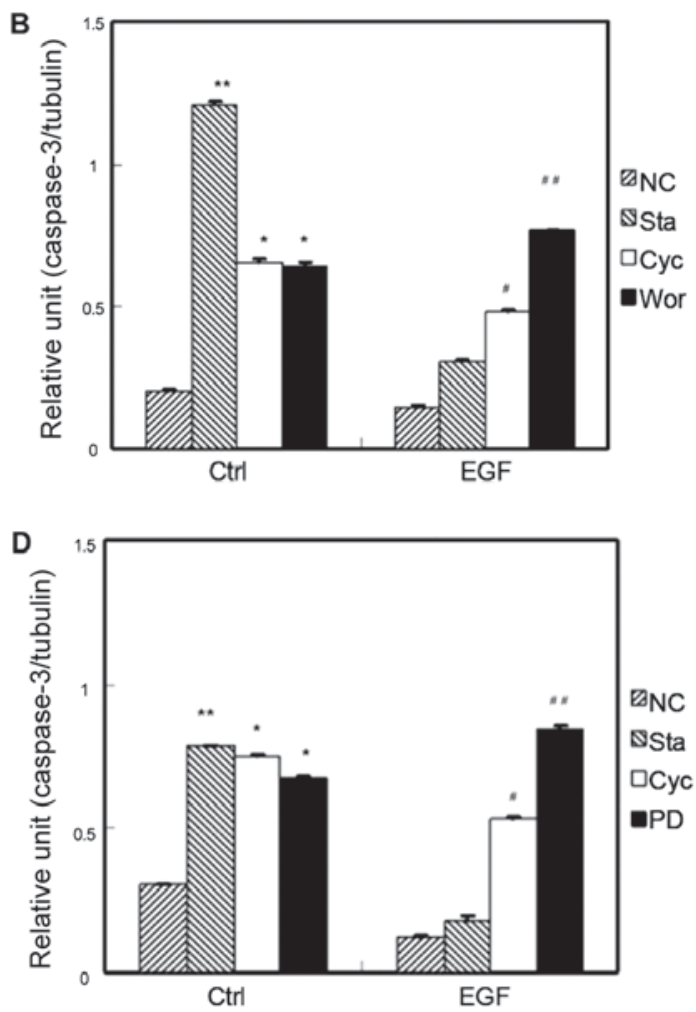

Figure 3. Effect of inhibiting Shh, PI3K and (or) MAPK signaling pathway on the expression of caspase-3 in GIST cells. (A) The influence on the expression of caspase-3 by inhibiting Shh and (or) PI3K signaling pathway in GIST cells. (B) The caspase-3/Tubulin relative intensity ratios of each treatment factor resulted from EGF-mediated cell apoptosis actions by inhibiting Shh and (or) PI3K signaling pathway. (C) The influence on the expression of caspase-3 by inhibiting Shh and (or) MAPK signaling pathway in GIST cells. (D) The caspase-3/Tubulin relative intensity ratios of each treatment factor resulted from EGF-mediated cell apoptosis actions by inhibiting Shh and (or) MAPK signaling pathway $\left({ }^{*} \mathrm{P}<0.05,{ }^{* *} \mathrm{P}<0.05\right.$ vs. ctrl-NC; ${ }^{\#} \mathrm{P}<0.05$, ${ }^{\# \#} \mathrm{P}<0.05$ vs. EGF-NC). N-Shh, recombinant Sonic hedgehog; GIST, gastrointestinal stromal tumor; EGF, endothelial growth factor; NC-normal control, Sta-starvation, Cyc-cyclopamine, PD-PD98059, Wor-wortmannin.

$(\mathrm{P}<0.05)$. The signal intensity of the $\mathrm{N}-$ Shh treatment group was significantly higher than that of the control group $(\mathrm{P}<0.05)$. In the treatment group, the signal intensity of the PD98059- and PD98059+CPN-KAAD-treated groups was significantly lower than that of the control group $(\mathrm{P}<0.05)$. The signal intensity of the CPN-treated group was slightly lower than that of the control group ( $\mathrm{P}<0.05$; Fig. $2 \mathrm{C}$ and $\mathrm{D})$.

In Gli-1 expression induced by N-Shh and interfered by the targeted drug of different signaling pathways, the signal intensity of the wortmannin-treated group was lower than that of the control group $(\mathrm{P}<0.05)$. The relative ratios of the grayscales of the CPN-KAAD- and wortmannin+CPN-KAAD-treated groups were lower than that of the control group $(\mathrm{P}<0.05)$. The signal intensity of the N-Shh-treated group was significantly higher than that of the non-N-Shh-treated group $(\mathrm{P}<0.05)$. In the N-Shh-treated group, the signal intensity of the drug-treated group was significantly lower than that of the N-Shh-induced control group $(\mathrm{P}<0.05)$. Regardless of $\mathrm{N}-\mathrm{Shh}$ treatment, the signal intensity of the co-treatment group was about lower than those of the wortmannin- and CPN-KAAD-treated groups $(\mathrm{P}<0.05)$ (Fig. 2E and $\mathrm{F}$ ). In Fig. $2 \mathrm{G}$ and $\mathrm{H}$, the signal intensity of the PD98059-treated group was slightly lower than that of the control group $(\mathrm{P}<0.05)$. The signal intensity of the CPN-KAAD- and PD98059+CPN-KAAD-treated groups was lower than that of the control group $(\mathrm{P}<0.05)$. The signal intensity of the N-Shh-treated group was significantly higher than that of the non-N-Shh-treated control group $(\mathrm{P}<0.05)$. In the
$\mathrm{N}$-Shh-treated group, the signal intensity of the drug-treated group was lower than that of the N-Shh-treated control group $(\mathrm{P}<0.05)$. Regardless of the N-Shh treatment, the signal intensityof the co-treatment group was significantly lower than those of the PD98059- and CPN-KAAD-treated groups $(\mathrm{P}<0.05)$.

Effects of Shh, PI3K and MAPK signaling inhibition on caspase-3 expression. Fig. 3 shows the effect of inhibitory Shh, PI3K, and MAPK signaling pathways on caspase-3 expression. With Shh and/or PI3K inhibition, after $12 \mathrm{~h}$ of serum starvation of GIST-H1 cells, the relative ratios of the grayscales of caspase-3 to tubulin were significantly higher than that of the negative control group $(\mathrm{P}<0.05)$. After $12 \mathrm{~h}$ of serum starvation of the GIST-H1 cells, the signal intensity of the CPN-KAAD- and wortmannin-treated groups was slightly lower than that of the starvation control group, but the difference was not significant $(\mathrm{P}>0.05)$. The signal intensity of the EGF-treated serum starvation group was slightly higher than that of the EGF-treated control group, but the difference was not significant $(P>0.05)$. The signal intensity of the EGF-treated wortmannin group was significantly higher than that of the control group $(\mathrm{P}<0.01)$. The signal intensity of the EGF- and CPN-KAAD-treated group was higher than that of the EGF-treated control group ( $\mathrm{P}<0.05$; Fig. $3 \mathrm{~A}$ and $\mathrm{B})$.

In Fig. 3C and D, with Shh and/or MAPK signaling pathway inhibition, after $12 \mathrm{~h}$ of serum starvation of the GIST-H1 cells, the signal intensity of caspase- 3 to tubulin was 
A

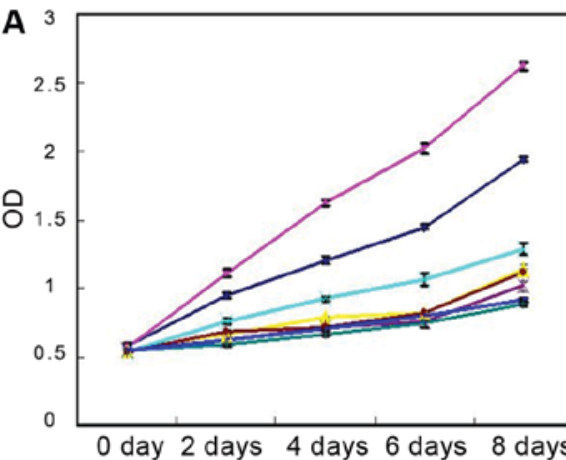

$\rightarrow$ Con

-EGF

$\rightarrow$ Cyc

$-\mathrm{EGF}+\mathrm{Cyc}$

*Wor

$\rightarrow$ EGF+PD

- Wor+Cyc

- EGF+Wor+Cyc
B

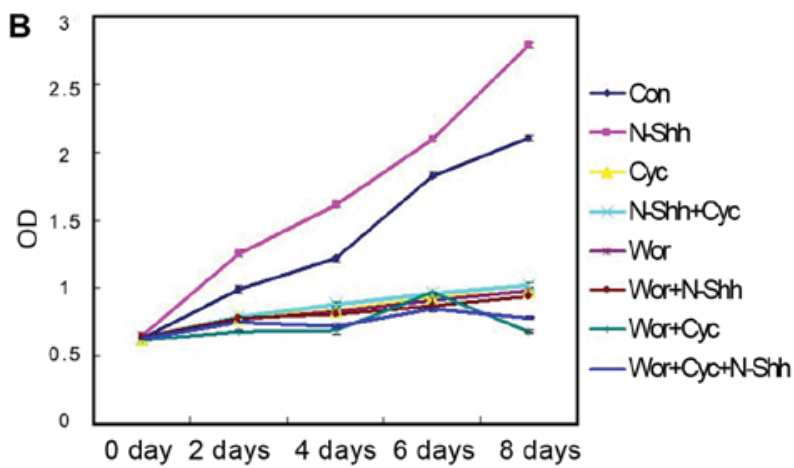

D



Figure 4. Effect of Shh on cell proliferation. (A) The effect on cell proliferation by inhibiting Shh and PI3K signals after activation of GIST-H1 cells with EGF. (B) The effect on cell proliferation by inhibiting Shh and PI3K signals after activation of GIST-H1 cells with N-Shh. (C) The effect on cell proliferation by inhibiting Shh and MAPK signals after activation of GIST-H1 cells with EGF. (D) The effect on cell proliferation by inhibiting Shh and MAPK signals after activation of GIST-H1 cells with N-Shh. OD, optical density; MAPK, mitogen-activated protein kinase; Shh, Sonic hedgehog; GIST, gastrointestinal stromal tumor; EGF, endothelial growth factor.

significantly higher than that of the negative control group $(\mathrm{P}<0.05)$. After $12 \mathrm{~h}$ of serum starvation of the GIST-H1 cells, the signal intensity of the CPN-KAAD- and PD-treated groups was slightly higher than that of the starvation control group $(\mathrm{P}<0.05)$. The signal intensity of the EGF-treated serum starvation group was slightly higher than that of the EGF-treated control group $(\mathrm{P}<0.05)$. The signal intensity of the EGF- and PD98059-treated group was significantly higher than that of the EGF-treated control group $(\mathrm{P}<0.01)$. The signal intensity of the EGF- and CPN-KAAD-treated group was higher than that of the EGF-treated control group ( $\mathrm{P}<0.05$; Fig. 3C and D).

Effects of Shh, PI3K, and MAPK signaling inhibition on cell proliferation. Fig. 4 shows the effect of inhibitory Shh, PI3K, and MAPK signaling pathways on cell proliferation. With Shh and PI3K signaling pathways inhibition, compaered with that in the blank control treatment, the proliferation rate of the GIST-H1 cells significantly increased under EGF treatment $(\mathrm{P}<0.01)$, and the effect can be partially blocked by wortmannin and CPN-KAAD pretreatment. Under N-shh treatment $(\mathrm{P}<0.01)$, the proliferation rate of the GIST-H1 cells also significantly increases $(\mathrm{P}<0.01)$, and the effect can be prevented by wortmannin and CPN-KAAD pretreatment (Fig. 4A and B). With Shh and MAPK signaling pathways inhibition, compared with that in the blank control treatment, the proliferation rate of the GIST-H1 cells significantly increased under EGF treatment, and the effect can be partially blocked by wortmannin and CPN-KAAD pretreatment (Fig. 4C). Under N-shh treatment, the proliferation rate of the GIST-H1 cells also significantly increased, but the effect can be evidently inhibited by PD98059 and CPN-KAAD pretreatment (Fig. 4D).

\section{Discussion}

Considering the important signal proteins of the three signaling pathways as targets, we believe that the development of targeted drugs will be one of the priorities in tumor therapy in the future.

The Shh signaling pathway is expressed in GIST tissues (10) and is associated with the risk rating of GIST. Detecting in GIST-H1 cell lines via western blot, we found that p-AKT, p-ERK, and Gli-1 are expressed in the three signaling pathways. In GIST-H1 cells, the expression of the PI3K/AKT and MAPK/ERK signaling pathways can be upregulated by the stimulation of their self-specific stimulatory factor EGF and by the stimulation of the stimulatory factor of the Shh signaling pathway. Conversely, the expression of the PI3K/AKT and MAPK/ERK signaling pathways can be inhibited by their self-specific inhibitor and by the specific inhibitor of the Shh signaling pathway. The combined use of the inhibitor (CPN-KAAD+wortmannin) of the Shh and PI3K/AKT signaling pathways and the inhibitor of the wortmannin+CPN-KAAD or PD98059+CPN-KAAD of the Shh signaling pathway can further partially block or suppress the expression of the two signaling pathways. Hence, the upregulation and inhibition of PI3K/and MAPK signaling pathways in GIST-H1 cells are related to the Shh signaling pathway.

In GIST-H1 cells, the expression of the Shh signaling pathway can be upregulated by the stimulation of its self-specific stimulatory factor N-Shh and by the stimulation of 
the stimulatory factor EGF of the PI3K/AKT and MAPK/ERK signaling pathways. The activity of the Shh signaling pathway can be inhibited by its self-specific inhibitor and by the specific inhibitor of the PI3K/AKT and MAPK/ERK signaling pathways. The combined use of the inhibitor (CPN-KAAD+wortmannin) of the Shh and PI3K/AKT signaling pathway or the inhibitor (CPN-KAAD+PD98059) of the Shh and MAPK/ERK signaling pathways can further inhibit its activity. Hence, the upregulation and inhibition of the Shh signaling pathway in GIST-H1 cells are related to PI3K/and MAPK signaling pathways.

Shh, PI, and MAPK signaling pathways can be activated in GIST and can be inhibited by their corresponding inhibitors. This result indicates that the three signaling pathways exist in GIST and that cross regulation occurs among these pathways.

The effect of inhibitory Shh, PI3K, and MAPK signaling pathways on the caspase-3 expression in GIST-H1 cells can be detected via western blot. Regardless of the stimulatory factors, the relative ratios of the grayscales of these signaling pathways are higher than that of the control group $(\mathrm{P}<0.05)$. This result suggests that specific Shh, PI3K, and MAPK signaling pathway inhibitors can accelerate GIST cell apoptosis. Proliferation experiment demonstrates that the proliferation rate of GIST-H1 cells significantly accelerates under EGF or N-shh treatment and that it can be partially inhibited by PD98059, CPN-KAAD, and wortmannin pretreatment. This result implies that the inhibitory Shh, PI3K, and MAPK signaling pathways can block the proliferation of GIST cells.

In summary, the data in GIST H1 cells confirmed that the combination of the above three inhibitors of the signal pathway can effectively inhibit the expression of GIST signaling and cell proliferation, and therefore can be considered as targeted therapy options in GIST.

\section{Acknowledgements}

Not applicable.

\section{Funding}

The present study received internal funding from the First Affiliated Hospital of Anhui Medical University.

\section{Availability of data and materials}

The datasets used and/or analyzed during the current study are available from the corresponding author on reasonable request.

\section{Authors' contributions}

YQ, WZ and XM contributed to the study design, experimental design and manuscript writing. ZW, QX, and JC contributed to the experimental design and manuscript writing.

\section{Ethics approval and consent to participate}

Not applicable.

\section{Patient consent for publication}

Not applicable.

\section{Competing interests}

The authors declare that they have no competing interests.

\section{References}

1. Foo WC, Liegl-Atzwanger B and Lazar AJ: Pathology of gastrointestinal stromal tumors. Clin Med Insights Pathol 5: 23-33, 2012.

2. DeMatteo RP, Lewis JJ, Leung D, Mudan SS, Woodruff JM and Brennan MF: Two hundred gastrointestinal stromal tumors: Recurrence patterns and prognostic factors for survival. Ann Surg 231: 51-58, 2000

3. Tsutsui S, Matsuyama A, Yamamoto M, Takeuchi H, Oshiro Y, Ishida T and Maehara Y: The Akt expression correlates with the VEGF-A and -C expression as well as the microvessel and lymphatic vessel density in breast cancer. Oncol Rep 23: 621-630, 2010.

4. Zhang H, Fagan DH, Zeng X, Freeman KT, Sachdev D and Yee D: Inhibition of cancer cell proliferation and metastasis by insulin receptor downregulation. Oncogene 29: 2517-2527, 2010.

5. Daniels M, Lurkin I, Pauli R, Erbstösser E, Hildebrandt U, Hellwig K, Zschille U, Lüders P, Krüger G, Knolle J, et al: Spectrum of KIT/PDGFRA/BRAF mutations and Phosphatidylinositol-3-Kinase pathway gene alterations in gastrointestinal stromal tumors (GIST). Cancer Lett 312: 43-54, 2011.

6. Wancket LM, Frazier WJ and Liu Y: Mitogen-activated protein kinase phosphatase (MKP)-1 in immunology, physiology and disease. Life Sci 90: 237-248, 2012.

7. Zhao C, Chen A, Jamieson CH, Fereshteh M, Abrahamsson A, Blum J, Kwon HY, Kim J, Chute JP, Rizzieri D, et al: Hedgehog signalling is essential for maintenance of cancer stem cells in myeloid leukaemia. Nature 458: 776-779, 2009.

8. Yoshizaki A, Nakayama T, Naito S, Wen CY and Sekine I: Expressions of sonic hedgehog, patched, smoothened and Gli-1 in human intestinal stromal tumors and their correlation with prognosis. World J Gastroenterol 12: 5687-5691, 2006.

9. Long B, Zhu H, Zhu C, Liu T and Meng W: Activation of the Hedgehog pathway in chronic myelogeneous leukemia patients. J Exp Clin Cancer Res 30: 8, 2011.

10. Qi Y,Zhao W, Wang Z and Meng X: Expression of Shh signaling pathway factors in gastrointestinal stromal tumor tissues and their associations with clinical pathological factors. Int J Clin Exp Pathol 9: 2841-2848, 2016. 